\title{
Nanotecnología: fuente de nuevos paradigmas
}

\author{
Luis Alberto Castañeda Naranjo* y Javier Palacios Neri **
}

RESUMEN: La innovación es un factor muy importante en el desarrollo humano. No obstante, pese a ser un aspecto del cual no se puede prescindir, debe estar acompañado de una política de responsabilidad social enfocada en el respeto por la sostenibilidad. Es necesario tomar en cuenta que la verdadera innovación está estrechamente relacionada con el correcto aprovechamiento de los recursos naturales y las materias primas. El desarrollo y la competitividad no deben servir como excusa para restar importancia al compromiso que sugieren los tiempos actuales. Por otro lado, el uso de las redes temáticas por parte de los grupos de investigación podrían llegar a convertirse en herramientas de suma importancia para la defensa de un mundo con innovación y armonía.

PALABRAS CLAVE: Innovación, responsabilidad social, trabajo colaborativo, redes temáticas, desarrollo humano, desarrollo sostenible, recursos naturales.

ABSTRACT: Innovation is an important factor in human development. However, despite being an aspect that cannot be avoided, must be accompanied by a policy of social responsibility focused on respect for sustainability. It is necessary to note that the real innovation is closely related to the proper use of natural resources and raw materials. The development and competitiveness should not be an excuse to downplay the commitments that suggest the current times. Furthermore, the use of thematic networks by research groups could become into important tools for the defense of a world with innovation and harmony.

KEYWORDS: Innovation, social responsibility, collaborative work, thematic networks, human development, sustainable development, natural resources.

\section{Introducción}

La nanotecnología es el estudio, diseño, creación, síntesis, manipulación y aplicación de materiales, aparatos y sistemas funcionales a través del control de la materia a nanoescala (un nanómetro es la billonésima parte de un metro), la cual ha venido a revolucionar los nuevos productos (Hullman, 2006). En otras palabras, es tecnología aplicada a la manipulación de átomos y moléculas para crear materiales y productos innovadores. Con el desarrollo del pensamiento científico, el ser humano ha logrado el control sobre los átomos y moléculas, y los expertos de la nanotecnología los acomodan en diferentes formas para producir nanomateriales con aplicaciones diversas.

\footnotetext{
* Maestro en economía y gestión de la innovación.

** Profesor titular de teoría económica. Departamento de Producción Económica. División de Ciencias Sociales y Humanidades. Universidad Autónoma Metropolitana, Campus Xochimilco.
} 
La nanotecnología adquiere relevancia y está presente en diferentes campos del conocimiento y en la vida diaria, atrae beneficios en diferentes industrias, sectores y toda la economía en su conjunto. En la actualidad, es un campo multidisciplinario con desarrollo a escala mundial. Existe un marcado interés por su desarrollo, su impacto positivo en la solución de problemas que con otras tecnologías es imposible solucionar.

Sus aplicaciones en campos diversos son notorios: en la industria automotriz para mejorar la resistencia de automóviles, en la informática es empleada en la creación de pequeños procesadores, en la industria textil ha servido para crear telas impermeables, aerodinámicas, ajustables para el uso deportivo (Hullman, 2006). Su desarrollo se muestra con éxito en la generación de materiales más resistentes que el acero; los equipos electrónicos cuentan ahora con conductores mejorados; en el campo de la informática se han generado dispositivos con mayor capacidad de almacenaje; en la medicina se han obtenido nuevos medicamentos para curar enfermedades sin dañar otras funciones del organismo y materiales de construcción inteligentes diseñados para el ahorro de energía, hacer más eficiente la generación de energía limpia como la solar, marina y eólica.

A nivel mundial se vive la experiencia de la nanotecnología para el desarrollo de fuentes de energía limpias y eficientes. El petróleo es una fuente de contaminación ambiental. En este sentido, muchos países consideran su remplazo por fuentes de energía sustentables. A pesar de que nuestro país ha basado gran parte de su economía en el petróleo, debe tomar acciones orientadas hacia la investigación de nuevas fuentes de energía y desempeñar un papel más activo en su desarrollo. La nanotecnología contribuye a detectar contaminantes en el ambiente o a remediar la contaminación del aire, agua y suelo.

Quienes operan en el campo de la nanotecnología sostienen la promesa de revolucionar un gran número de productos, procesos y aplicaciones. Prometen crear investigaciones interdisciplinarias y el desarrollo de plataformas de Investigación y Desarrollo (IyD) para la industria de la medicina, y direccionar aplicaciones radicales como la detección y tratamiento de enfermedades, el eficiente monitoreo y protección del ambiente, producir y almacenar energía y construir una compleja estructura de los circuitos electrónicos para los aviones (Huang et al., 2005).

El impulso de la nanotecnología ha permitido el uso eficiente de los recursos materiales; también ha generado un impacto sobre las telecomunicaciones; en cuanto a la biomedicina, ayuda a descifrar la naturaleza a escala del DNA; en la instrumentación médica, los medicamentos y su efectividad.

Es por estos avances de la nanotecnología que es reconocida por más de sesenta países como un elemento central para el desarrollo de sus economías (Huang et al., 2005). Además, para todas las tecnologías, incluida la nanotecnología, el ser humano tiene la responsabilidad de actuar de manera ética y de aplicarla para beneficio de la sociedad. Por ello, los científicos trabajan con 
precisión y sobre bases científicas estrictas para estudiar a profundidad las propiedades de los nanomateriales y determinar los posibles riesgos a corto, mediano y largo plazo.

Este trabajo se encuentra estructurado de la siguiente manera: como primera instancia se plantean la metodología, es decir, cómo se procedió en la búsqueda y manipulación de la información de la tecnología. A continuación, se analiza el patentamiento de la nanotecnología con clasificación B82 en el periodo 1976-2011. La siguiente sección hace referencia a la clasificación que Lux Research realiza a los países que se han dedicado a la investigación y explotación de la nanotecnología y se comenta la importancia que tiene el papel del estado para invertir en IyD en apoyo a la tecnología. La última parte corresponde a las conclusiones de la evolución del patentamiento y se resumen los resultados más relevantes. Es necesario mencionar que para el análisis sólo se presentarán las gráficas con el fin de ilustrar el comportamiento de la nanotecnología, de tal modo que la presentación sea más didáctica. Se incorpora, además, un apartado de anexos.

\section{Objetivos}

Analizar la trayectoria de la nanotecnología en el periodo que va de 1976 a 2011, tomando las patentes otorgadas por la United States Patent and Trademark Office (USPTO). Resulta necesario el análisis, evaluación y debate sobre las implicaciones de esta tecnología y futuras implicaciones en otras aéreas.

\section{Metodología}

Este trabajo está basado en la clasificación de la World Intellectual Property Organization (WIPO), la cual proporciona la clasificación internacional de la tecnología en estudio: (Nanotecnología B82) con esta clasificación, se accedió al sitio web de la USPTO para buscar la tecnología a través de queries diseñados para la búsqueda. Al introducir la clasificación correspondiente, USPTO refiere una base de 318 patentes de las cuales se seleccionaron cada uno de los siguientes elementos: i) clasificación internacional (CIP); ii) el concesionario (assigne); iii) el país; iv) los inventores; v) la fecha de solicitud, de entrega, de prioridad, y, por último, vi) descripción de la patente. Con estos elementos se elabora el análisis para acentuar la relevancia y mostrar la trayectoria de la nanotecnología.

\section{Antecedentes}

Existe un número limitado de referencias para corroborar la protección a la creatividad. Aunque la práctica es muy antigua, sus orígenes modernos la ubican a finales del siglo XVIII sobre el reconocimiento a indicaciones de tipo 
CUADRO 1. Clasificación de la nanotecnología.

\begin{tabular}{|l|l|}
\hline CLASIFICACIÓN & DESCRIPCIÓN \\
\hline Sección B & $\begin{array}{l}\text { Desempeño operacional en transporte. } \\
\text { Microestructura tecnológica, nanotecnología. }\end{array}$ \\
\hline B82 & \begin{tabular}{l} 
Nanotecnología. \\
\hline Nanotecnología
\end{tabular} \\
$\begin{array}{l}\text { En esta clasificación se utilizan los siguientes términos con } \\
\text { el siguiente significado: } \\
\text { "Nanotamaño" o "nanoescala" se refiere a un tamaño } \\
\text { controlado geométricamente por debajo de los 100 } \\
\text { nanometros (nm) en una o más dimensiones. } \\
\text { Nanoestructuras es una entidad con al menos un solo } \\
\text { nanocomponente funcional que hace propiedades } \\
\text { físicas, químicas o biológicas con efectos disponibles } \\
\text { a nanoescala. }\end{array}$ \\
\hline B82B & $\begin{array}{l}\text { Nanoestructuras formadas por manipulación de átomos } \\
\text { individuales, moléculas o colección limitada de átomos } \\
\text { o moléculas como unidades discretas, fabricación o } \\
\text { tratamiento del mismo. }\end{array}$ \\
\hline B82Y & $\begin{array}{l}\text { Usos, aplicaciones, tratamientos y fabricación de } \\
\text { nanoestructuras especificas. }\end{array}$ \\
\hline
\end{tabular}

Fuente: World Intellectual Property Organisation, WIPO 2011.

geográfico en Francia. Se tiene información, sin embargo, de que en el siglo XV, Fillipo Brunellesqui solicitó, en la ciudad de Florencia, un privilegio temporal para usar por tres años de manera exclusiva, el diseño de una barca para transportar mármol a la catedral de la ciudad. Aun más, en plena era del Renacimiento en el siglo XVI, Galileo Galilei solicitó el derecho de hacer y usar en forma exclusiva su máquina para llevar agua para irrigar sus tierras altas con pequeño gasto y gran conveniencia para sus propósitos personales pues le había costado mucho trabajo y consideraba injusto que se reconociera como propiedad común.

Con el advenimiento de la Revolución industrial y en particular, en Inglaterra, se encuentra documentado el uso de la palabra patente para determinados derechos reales adquiridos a los que se les impondrían criterios estrictos para su otorgamiento y para su vigencia y explotación en determinado tiempo. Con el surgimiento de la imprenta se hizo realidad la protección de creaciones artísticas y literarias pues además favoreció la divulgación y propagación de las obras impresas en forma masiva. Un estatuto de la reina Ana de Inglaterra promulga que la titularidad exclusiva para del editor al autor, otorgándose a éste y a sus derechohabientes el derecho exclusivo de impresión y reimpresión.

No existió en todo ese tiempo una protección efectiva y sistemática que permitiera a nivel jurídico, la protección de las ideas y creaciones de artistas e inventores. Se ignora cuánto recibieron los grandes músicos por sus obras, 
cuánto por la vacuna contra la rabia. Puede ser que hayan recibido una paga escasa o ninguna sobre sus aportaciones. Con la extensión general de las relaciones de producción capitalista se hace más amplio su uso y se extiende con la producción en serie y la caracterización capitalista del mercado donde todo lo que se produce, crea o inventa, se convierte en mercancía para la obtención de plusvalía.

El proceso de constitución del derecho de propiedad ha sido contradictorio y siempre con dificultades cuando se refiere a invenciones e innovaciones técnicas, creaciones artísticas y literarias. Sin embargo, se piensa que el proceso por el cual la propiedad intelectual se constituyó en una institución jurídica inició con los derechos otorgados por algunos Estados europeos dentro de sus fronteras. En la actualidad, cuando se habla de propiedad intelectual se hace referencia a la protección jurídica de la creatividad del conjunto de invenciones, distinciones comerciales, expresiones artísticas y literarias protegidas por el Estado. Pero no todo producto del intelecto humano es susceptible de protección jurídica como propiedad intelectual. Algunas ideas para convertirse en sujetos de protección jurídica deben contar con un soporte material y demostrar su utilidad o beneficio para la sociedad.

Por eso, las normas de protección jurídica tienden a establecer fronteras en ocasiones difusas y en otras diferenciadas entre las diversas figuras que adquiere la protección, tomando como base la utilidad técnica y comercial o la forma de expresión artística. En su sentido más amplio, la propiedad intelectual comprende a la propiedad industrial y los derechos de autor, éstos, a su vez, son conocidos como propiedad intelectual en sentido estricto por diversas legislaciones en cada país o Estado.

En México, el primer ordenamiento jurídico en materia de propiedad industrial relacionado con invenciones fue el Decreto de las Cortes Españolas, expedido el 2 de octubre de 1820. En 1832, aparece la primera ley mexicana en la materia llamada Ley Sobre el Derecho de Propiedad de los Inventores o Perfeccionadores de algún Ramo de la Industria. En relación con las marcas, la primera reglamentación en la materia se plasmó en los artículos 1418 y 1423 del Código de Comercio, promulgado en 1884, con el propósito de proteger el nombre del comerciante o prestador de servicios.

Se considera la propiedad industrial como derecho patrimonial de carácter exclusivo, otorgado por el Estado mexicano por un tiempo determinado a la persona física o moral que realiza invenciones o innovaciones como un producto técnico nuevo, una mejora a una máquina o aparato, un diseño original para hacer más útil o atractivo un producto o un proceso de fabricación novedoso, o bien, a aquellas personas que adoptan indicaciones comerciales para distinguir sus productos o servicios de otros de su misma especie en el mercado. Así, un producto nuevo a nivel técnico, una mejora, un diseño original o un diseño de fabricación novedoso comprende al grupo de las invenciones a proteger mediante la solicitud y expedición de una patente, un modelo de utilidad o un diseño industrial, así como a través de los secretos 
industriales. La adopción de indicaciones comerciales para distinguir los productos y servicios de otros de su misma especie, se refiere a signos distintivos protegidos por medio de registros de marcas y avisos comerciales, publicación de nombres comerciales y declaración de denominación de origen.

La propiedad intelectual se constituye a partir de un conjunto de normas que regulan la existencia de prerrogativas y beneficios que las leyes reconocen y establecen para estas figuras jurídicas a favor de sus creadores. El sistema de patentes fomenta el desarrollo tecnológico mediante la protección jurídica de quien ostenta los derechos sobre la invención o innovación tecnológica y permite a sus inventores obtener títulos de propiedad con carácter exclusivo y por un tiempo determinado, permite, además, a los investigadores, la posibilidad de acceder a información novedosa.

La invención se considera, por tanto, como la que ofrece bases para la innovación y es ésta la que en la práctica incide de manera notable en la eficiencia industrial, la productividad y el desarrollo económico. Por eso, esta concepción del sistema de patentes se encuentra considerada en la legislación mexicana después de un proceso de desarrollo social y como resultados de profundos análisis sobre los alcances de conceptos básicos en materia de propiedad industrial como privilegio, monopolio temporal, vigencia legal, demostración de la técnica, derechos de oposición, entre otros.

La Ley de Fomento y Protección de la Propiedad Intelectual entró en vigor el 21 de julio de 1991 y su reglamento se dio a conocer en 1994. Esta ley definió las bases para las actividades industriales y comerciales y perfeccionar sus procesos productivos, impulso al mejoramiento de la calidad de bienes y servicios conforme a intereses de consumidores y se ofreció un marco legal a la propiedad intelectual. Esta ley propició certidumbre jurídica a la inversión extranjera y transferencia de tecnología. Se anticipó a las nuevas tendencias internacionales en estos campos y abrió la oportunidad para el patentamiento a todas las áreas del conocimiento, con independencia de los campos tecnológicos al que pertenecían pero con mayor énfasis a aquellos como la biotecnología, los medicamentos, bebidas y alimentos para el consumo humano y animal, fertilizantes, pesticidas, entre otros. Amplió los periodos de protección de las patentes de 14 a 20 años a partir de la fecha legal de presentación de la solicitud. Para las marcas, fue de cinco a diez años. Se protegió la información técnica de naturaleza confidencial por medio del secreto industrial y se introdujo la figura del modelo de utilidad y el concepto de marca tridimensional. La ley desregulaba la transferencia de tecnología pues dejaba en manos de particulares su negociación y contratación. La ley fue considerada como única en su tipo a nivel mundial.

A partir de 1994, esta ley se conoce como Ley de Propiedad Intelectual. Entre sus reformas se encuentran las siguientes: perfecciona el sistema de propiedad intelectual con el uso y aplicación de medidas de simplificación administrativa, para facilitar el trámite y otorgamiento de derechos; establece un sistema más eficiente para sancionar a los infractores; se ajusta a 
otras disposiciones contenidas en leyes de otras naciones y fortalece la infraestructura internacional.

En la actualidad, las cosas son distintas. En los últimos años se ha producido un viraje interesante para el análisis. El patentamiento ha cambiado de manera sustancial desde las actividades de innovación e investigación privadas hacia las realizadas por las universidades y centros de enseñanza superior. En el aspecto comercial, las cosas no son distintas. Las patentes son fuente de ingresos futuros. Una garantía del negocio y la mayoría de las veces, una defensa contra los competidores. En este mundo globalizado y complejo, conocer cuáles son las empresas con mayor cantidad de patentes registradas permite saber ante quién se enfrentan los innovadores e investigadores.

Un caso especial y digno de estudio es el de Apple, la empresa vendedora del mayor número de teléfonos celulares. A diferencia de Apple, Samsung, Nokia, Alcatel-Lucent, IBM, Ericsson, Microsoft, Sony y Motorola, por ejemplo, son quienes mayor número de patentes tienen registradas. Google se encuentra incluso más allá de la posición de Apple. ¿Cómo es esto? Apple puede tener 15 patentes para elaborar un cable, es decir, muchas veces las patentes son para detalles concretos y, por tanto, importa la calidad en la estrategia del patentamiento, no la cantidad. Tal vez es esa manera de pensar de sus directivos las que hacen distinta la actuación en el mundo globalizado.

Apple tiene escasas patentes registradas sólo que son de calidad excepcional, suficiente para proteger a la empresa y para continuar como uno de los vendedores de artículos novedosos más dinámicos en la actualidad. Empresas como Samsung han seguido el camino opuesto: la cantidad como estrategia para conseguir lo mismo, patentar todo lo que se encuentre a su alcance por si algo hiciera falta en el futuro. Conviene señalar que Samsung produce un mayor número de bienes que Apple, pues entre su producción se puede encontrar desde televisores hasta estufas para cocinar, desde computadoras de todo tipo, incluyendo las novedosas tablets, hasta teléfonos celulares. Es probable que esta variedad de bienes le permita conseguir muchas más patentes en el futuro.

\section{Análisis de la patente}

Utilizando la metodología descrita líneas antes, la gráfica 1 muestra la trayectoria seguida por las invenciones de nanotecnología en el periodo que va de 1976 a 2011.

A lo largo del periodo de estudio, las invenciones en el campo de la nanotecnología muestran una tendencia ascendente. Esta proyección se debe a que la nanotecnología ha tenido a lo largo del tiempo, un sustancial impacto en el mundo económico a través de nuevas invenciones en diversas industrias. Sin embargo, esta tecnología en particular no corresponde a una industria que pueda ser identificable o cuantificable de manera fácil y sencilla. 
GRÁFICA 1. Trayectoria de la nanotecnología 1976-2011.

Nanotecnología: número de patentes otorgado en USPTO (1976-2011)

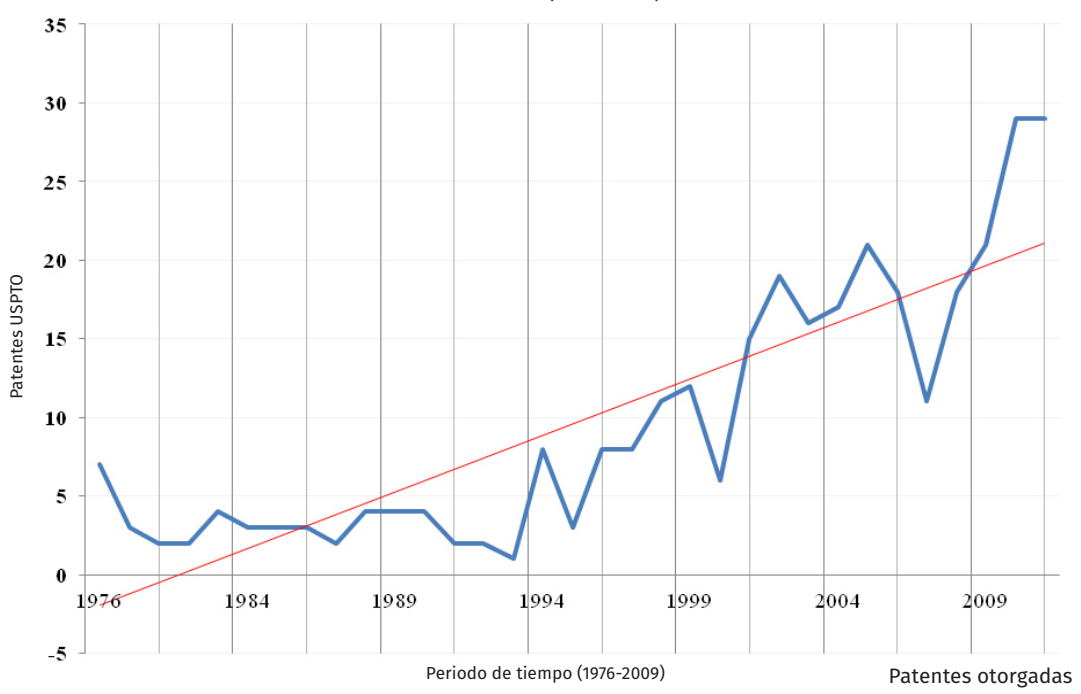

Fuente: Elaborada con información de USPTO, 2011.

Esta tecnología contribuirá de manera viable y exitosa a la mejora de productos existentes y a la generación de productos más sofisticados cuya elaboración requerirá de capital humano más especializado.

En el periodo de análisis se tiene una media de nueve patentes anuales otorgadas, mostrando para los primeros años, 1976-1994, un promedio de tres patentes otorgadas y a partir de $1995 \mathrm{y}$ hasta el final del periodo un promedio de patentamiento de 15 patentes anuales. Esto se debe a que más del $50 \%$ de las patentes otorgadas en el segundo periodo son desarrollos de Estados Unidos, quien registra ocho patentes por año en el periodo y continúa con su repunte a lo largo del estudio.

La tendencia del patentamiento de la nanotecnología en el periodo 1974 a 2011 también se puede observar en la gráfica 2, la cual representa un promedio de tres años de espera entre la fecha de solicitud y la fecha de otorgamiento. Éste es un factor relevante pues indica el tiempo en el cual la oficina de patentes americana revisa los diversos factores como la novedad inventiva, antes de asignar el derecho de propiedad al concesionario. Se puede acotar que el tiempo entre la fecha de solicitud y la fecha de otorgamiento es corto, pues existen tecnologías para las cuales este periodo es demasiado largo.

Una de las razones por las que es importante que el periodo de otorgamiento sea corto, es que genera incentivos a las firmas para seguir inventando, pues una vez que la patente es otorgada, las empresas reafirman su 
derecho para explotar la invención y proporcionar al mercado un producto que si es aceptado por los consumidores se pueda convertir en una innovación.

De la misma gráfica destacan algunos años por ejemplo, 1974, 1975, 1979 y 1980, donde no hubo otorgamiento de patentes en nanotecnología y para los años 1993 y 1998 se podría asumir que la cantidad de solicitud coincide con la misma cantidad de otorgamiento. No obstante, para completar esta gráfica es necesario la incorporación de todas las solicitudes rechazadas por diversos motivos. Sería más completa para el análisis de la tecnología si se contara con datos de cuántas de éstas fueron otorgadas por USPTO y se han convertido en innovación exitosa y otra línea que mostrara cuáles han servido para la creación de nuevo conocimiento.

La gráfica 3 permite observar los siete países que dominan el patentamiento en la rama bajo estudio. Estados Unidos se encuentra a la cabeza con la mayor cantidad de patentes de la tecnología en estudio. Japón le precede patentando $47.8 \%$. El predominio de Estados Unidos sobre los demás países en nanotecnología se debe a sus programas apoyados con financiamiento público por la National Science Foundation y Nanoscale Science and Engi-

GRÁFICA 2. Brecha entre la fecha de solicitud y la fecha de otorgamiento periodo (1974-2011).

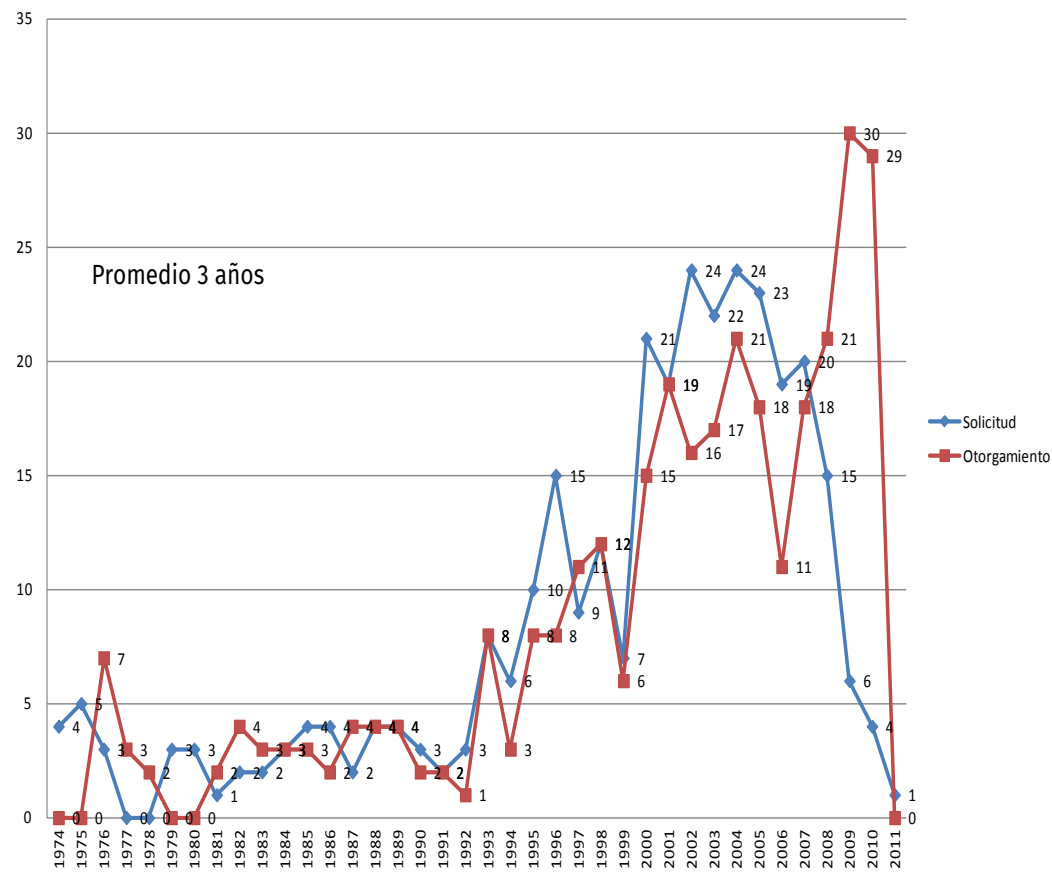

Fuente: Elaborada con información de USPTO, 2011. 
GRÁFICA 3. Patentes otorgadas en USPTO por países 1976-2011.

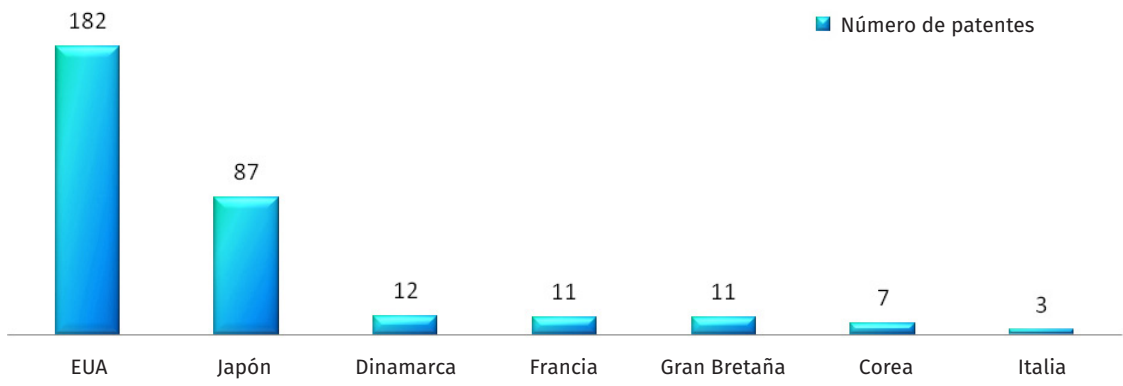

Fuente: Elaboración propia con base en USPTO, 2011.

neering, lo cual le permite desarrollar nuevos productos y procesos. Este apoyo es de alrededor del 20\% de los fondos para la educación en investigación básica (Huang et al., 2005). Estos programas de apoyo a la educación en ciencias e ingenierías generan premios y otorgan concesiones, contratos y acuerdos de cooperación a favor de la nanotecnología. Este tipo de programas están subdivididos en 638 subprogramas que incluyen el arduo trabajo de alrededor de 81,040 investigadores (Huang et al., 2005). Estudios como el de Lux Research mencionan que el predominio en la nanotecnología por parte de Estados Unidos se debe a que para el año 2009, este país invirtió 6.4 millones en gasto corporativo e inversión de capital de riesgo para el desarrollo de la nanotecnología (Lux Research, 2010).

La proporción entre países en el patentamiento de la nanotecnología se muestra en la gráfica 4. Se observa que Estados Unidos tiene más del 50\% de las patentes seguido por Japón con un 27.1\% del patentamiento. Países

GRÁFICA 4. Porcentaje de patentes otorgadas por principales países de USPTO.

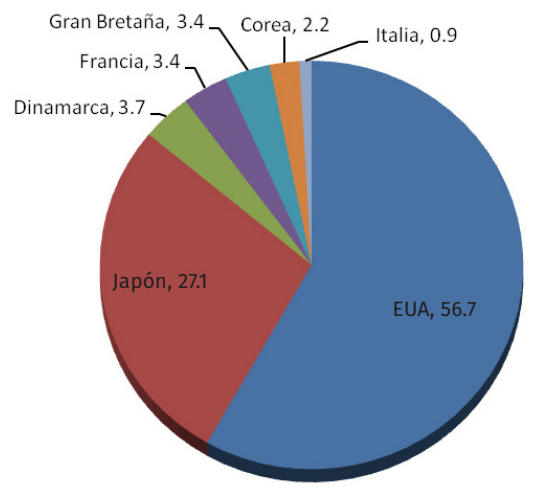

Fuente: Elaboración propia con base en USPTO, 2011. 
GRÁFICA 5. Nanotecnología: patentes otorgadas en USPTO principales asignaciones (1976-2011).

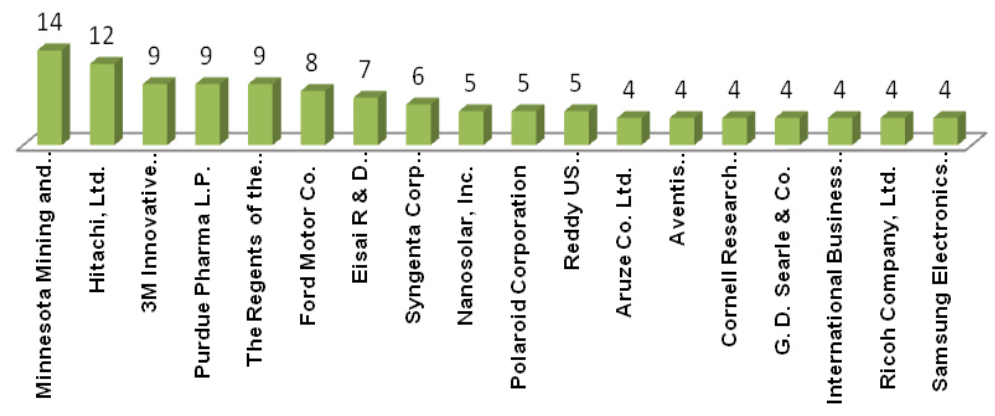

Fuente: Elaboración propia con base en USPTO, 2011.

como Dinamarca, Francia y Gran Bretaña hacen esfuerzos para posicionarse con un mayor número de patentes en la evolución de la tecnología.

El dinamismo del patentamiento se observa en la gráfica anterior a través de los principales concesionarios de patentes de nanotecnología en el periodo 1976-2011. Destacan las tres empresas más relevantes en la gráfica. En primer lugar, la empresa Minnesota Mining and Manufacturing (3M) la cual tiene presencia en 60 países y grandes inversiones en la industria electrónica. En segundo lugar se encuentra la empresa nipona Hitachi, LTd la cual, debido a su incursión en las tecnologías de la información, la electrónica, aparatos médicos y el medio ambiente, ha requerido de la investigación y desarrollo de nuevos productos a escala nano. En tercer lugar se encuentra 3M Innovative Properties, filial de 3M, la cual diversifica su tecnología e incursiona con productos para el cuidado del ambiente y prevenir la contaminación. Dada la naturaleza de estas empresas, en los últimos años han apostado de forma abierta y estratégica a la nanotecnología, y es por ello que se muestran como las principales patentadoras de dicha tecnología.

La gráfica 6 fue tomada del análisis de Lux Research que clasifica a los países por su actividad en nanotecnología. Destacan las siguientes definiciones para la muestra considerada por la empresa:

- Dominante: Países que ocupan el cuadrante superior derecho. Estos países cuentan con alta actividad en nanotecnología y el desarrollo de la fuerza tecnológica necesaria para su comercialización.

- Torre de marfil: Países en el cuadrante superior izquierdo. Éstos tienen alta actividad nanotecnológica, pero son menos propensos a desarrollar sus economías sobre la base de esta tecnología, debido a que tienen relativamente menores esfuerzos en el desarrollo tecnológico.

- Nicho: Países del cuadrante inferior derecho. Son potencias en el desarrollo de tecnologías altas y actividad en su desarrollo, pero sin escala para apoyar la competitividad internacional en nanotecnología. 
GRÁFICA 6. Agrupación por países por su actividad en nanotecnología

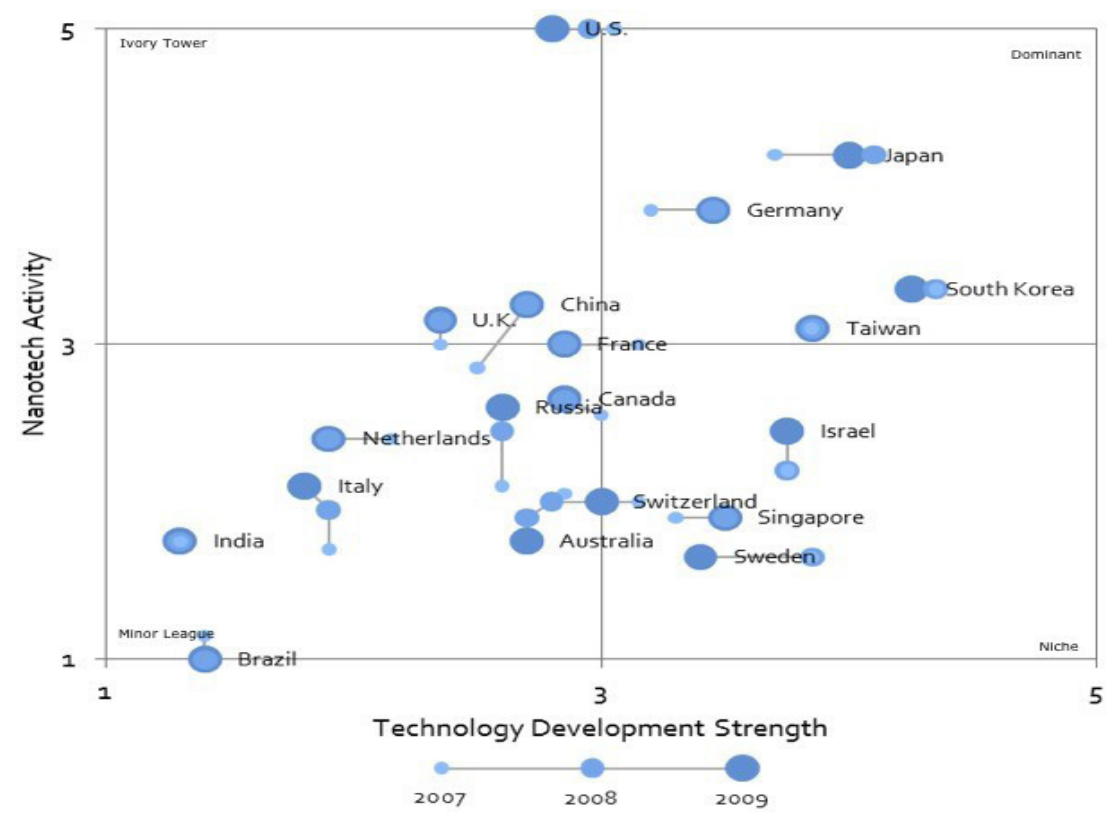

Fuente: Tomado de Lux Research, 2010.

Como resultado, estos países se centran en el desarrollo de centros de competencia con dominios específicos como la electrónica.

- Liga menor: Países ubicados en el cuadrante inferior izquierdo. Tienen una baja actividad de investigación y desarrollo lo cual se refleja en la baja actividad de investigación y comercialización de nanotecnología.

Como corolario puede observarse, en la gráfica 6, la participación nula de países de América Latina, aun cuando existe una base científica y tecnológica importante para el desarrollo y estudio de las nanociencias y nanotecnologías.

La gráfica 7 muestra el porcentaje de ventas de productos con nanotecnología incorporada en algunos de sus elementos. Como se observa, Asia, a pesar de no ser líder en el patentamiento de nanotecnología, incorpora mayor número de componentes a escala nano en los productos que vende.

La gráfica 8 permite comprobar, con el análisis de la evolución de nanotecnología, que los países con un número mayor de compañías, universidades y centros de investigación son los mismos que están en la frontera del patentamiento, tal es el caso de Estados Unidos que cuenta con el mayor número de patentes (182) seguido por Japón con 87 patentes. La relevancia de tener compañías e instituciones en investigación y desarrollo en tecnología se ve reflejada en la cantidad de patentamiento. 
GRÁFICA 7. Ventas globales de productos con nanotecnología incorporada. Estimación en porcentaje.

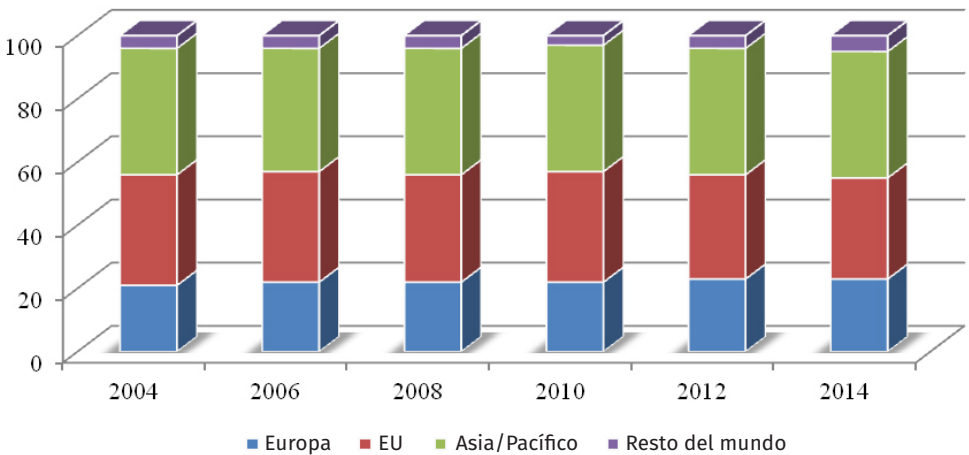

Fuente: Tomado de Lux Research, 2004.

La gráfica 9 ilustra la estimación en millones de euros $(1 €=1 \$)$ de fondos privados y públicos para la investigación y el desarrollo de nanotecnología en 2005, por principales países. Una vez más se corrobora el dominio de Estados Unidos como el país con mayor número de recursos en IyD para la realización de actividades en nanotecnología, al igual que la Unión Europea, cuenta con tres tipos de fondos: federal, estatal y privado. Esto le permite incursionar en las nuevas técnicas y en los conocimientos de frontera para poder inventar y patentar nanotecnología.

GRÁFICA 8. Compañías, universidades y centros de investigación que realizan actividades de nanotecnología, por países.

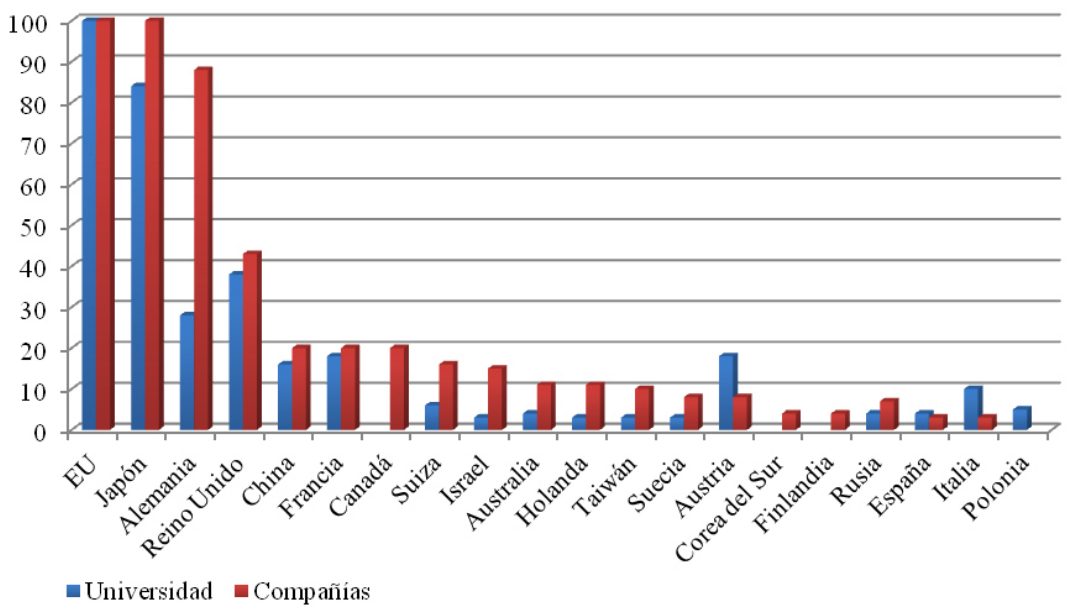

Fuente: Elaborado con datos de Científica, 2003. Para Estados Unidos, las empresas son 420, la gráfica fue ajustada. 
GRÁFICA 9. Estimación de los fondos públicos para la investigación de IyD en 2004 por principales países, 2003 (miles de euros).

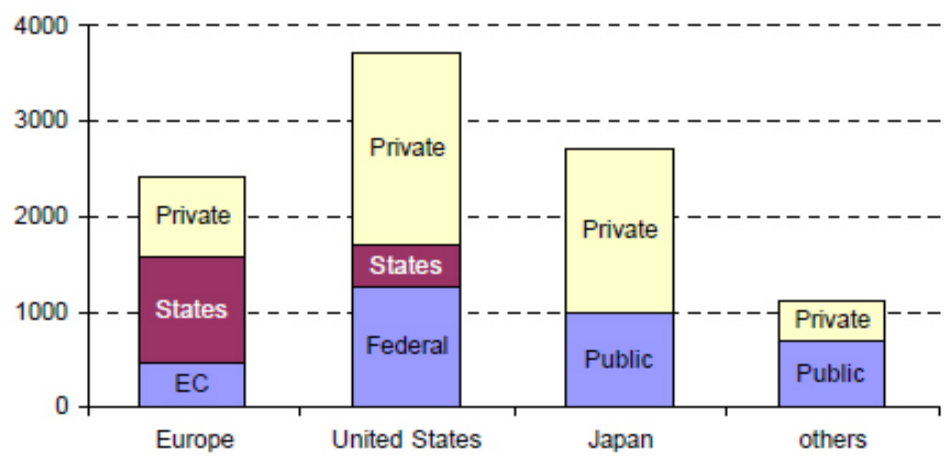

Fuente: Comisión Europea, 2005.

\section{Conclusiones}

La evolución de las patentes de nanotecnología en el periodo 1976-2011 muestra un comportamiento ascendente aunque moderado; sin embargo, en los últimos años ha obtenido mayor relevancia la nanotecnología pues a pesar de no estar clasificada como una industria específica ha empezado a revolucionar algunas industrias trayendo grandes beneficios por su incorporación.

Un aspecto importante por mencionar es el hecho de que el promedio de espera del otorgamiento de la patente en nanotecnología es aproximadamente de tres años. Ésta es una característica positiva de esta tecnología pues existen tecnologías cuyo periodo de patentamiento es muy largo.

Existe una concentración elevada de esta tecnología en Estados Unidos y Japón. Son éstos los que cuentan con un número mayor de patentes y permite asumir su especialización en la investigación y el desarrollo de nanotecnología, y que desean incursionar cada vez más en el estudio de esta tecnología para la elaboración de productos diferenciados.

Cabe señalar que a nivel mundial, los países que tienen mayor número de patentes coinciden con los que tienen un mayor número de compañías, universidades y centros públicos de investigación. Asimismo, coincide que aquellos países con altos montos de presupuesto para la investigación y desarrollo son los mismos países como es el caso de Estados Unidos que cuenta con fondos federales, estatales y privados para la actividad en nanotecnología.

Dado estos resultados, se recomienda concentrar la atención en este tipo de tecnologías, las cuales rompen paradigmas y serán las que revolucionen las industrias y los sectores en los países. 
Un análisis más exhaustivo sobre la relevancia de nanotecnología para México sería de gran importancia, pues se podría recomendar a los hacedores de políticas públicas un mayor apoyo en IyD en actividades de nanotecnología, a modo que sea un respiro para las industrias en nuestra economía.

\section{Anexos}

TABLA 1. Patentes otorgadas, principales países.

\begin{tabular}{|l|c|c|}
\hline USPTO: PATENTES OTORGADAS POR PAIS & $\mathbf{1 9 7 6}-\mathbf{2 0 1 1}$ \\
\hline USA & 182 & 56.7 \\
\hline Japón & 87 & 27.1 \\
\hline Dinamarca & 12 & 3.7 \\
\hline Francia & 11 & 3.4 \\
\hline Reino Unido & 11 & 3.4 \\
\hline Corea & 7 & 2.1 \\
\hline Italia & 3 & 0.9 \\
\hline Taiwán & 3 & 0.9 \\
\hline Austria & 1 & 0 \\
\hline Bélgica & 1 & 0 \\
\hline Israel & 1 & 0 \\
\hline Luxemburgo & 1 & 0 \\
\hline
\end{tabular}

Fuente: Elaboración propia con base en USPTO, 2011.

TABLA 2. Patentes por principales asignaciones.

\begin{tabular}{|l|l|}
\hline ASIGNACIONES PRINCIPALES & 14 \\
\hline Minnesota Mining \& Manufacturing Co. & 12 \\
\hline Hitachi, Ltd. & 9 \\
\hline 3M Innovative Properties Co. & 9 \\
\hline Purdue Pharma, L. P. & 9 \\
\hline The Regents of the University of California & 8 \\
\hline Ford Motor, Co. & 7 \\
\hline Eisai R\&D Management Co. Ltd. & 6 \\
\hline Syngenta Corp Protection, Inc. & 5 \\
\hline Nanosolar, Inc. & 5 \\
\hline Polaroid Corporation & 5 \\
\hline Reddy US Therapeutics, Inc. & 4 \\
\hline Aruze Co. Ltd. & \\
\hline
\end{tabular}

Fuente: Elaboración propia con base en USPTO, 2011. 
TABLA 3. Fondos públicos en $1000 €$, para la investigación de lyD en 2005 por principales países $€(1 €=1 \$)$.

\begin{tabular}{|l|l|}
\hline EUA (Federal) & 910,000 \\
\hline Japón & 750,000 \\
\hline Comisión Europea & 370,000 \\
\hline EUA (Estados) & 333,300 \\
\hline Alemania & 293,100 \\
\hline Francia & 223,900 \\
\hline Corea del Sur & 173,300 \\
\hline Reino Unido & 133,000 \\
\hline China & 83,300 \\
\hline Taiwán & 75,900 \\
\hline
\end{tabular}

Fuente: Comisión Europea, 2005.

Primera patente otorgada por USPTO de la clasificación B82 (nanotecnología).

\section{United States Patent [19]}

\section{Dickie et al.}

[11]

$3,957,918$

[45] May 18, 1976
[54] RADIATION POLYMERIZABLE COATING COMPOSITION CONTAINING AN UNSATURATED PHOSPHORIC ESTER

[75] Inventors: Ray A. Dickie, Birmingham; Joseph C. Cassatta, Taylor, both of Mich.

[73] Assignee: Ford Motor Company, Dearborn, Mich.

[22] Filed: Dec. 31, 1974

[21] Appl. No.: 537,736

[52] U.S. Cl.. 260/885; 204/159.15 204/159.16; 260/836; 260/837 R; 260/859 R $260 / 886 ; 260 / 953 ; 427 / 44 ; 427 / 404$ $427 / 407 ; 427 / 250 ; 428 / 442 ; 428 / 463$ $428 / 515 ; 428 / 537$

[51] Int. $\mathrm{Cl}^{2}$. C08L 31/02; C08F 8/00

[58] Field of Search............... 204/159.15, 159.16, 204/159.17; 260/953, 461, 885, 886; 427/44

\begin{tabular}{|c|c|}
\hline \multicolumn{2}{|c|}{$\begin{array}{l}\quad \text { References Cited } \\
\text { UNITED STATES PATENTS }\end{array}$} \\
\hline $4 / 1960$ & O'Brien et al. \\
\hline $1 / 1972$ & Pinkney et al.................260/86.1 260.28 \\
\hline $1 / 1974$ & Rees et al. ..................... 260/41 A \\
\hline
\end{tabular}

\section{OTHER PUBLICATIONS}

Gotoda et al., Radiation Curing of Mixtures of Diallylphthalate Prepolymer and Vinyl Monomer, pp. 121-129 (1970).
Primary Examiner-Richard B. Turer Attorney, Agent, or Firm-Roger L. May; Keith L. Zerschling

\section{[57]}

\section{ABSTRACT}

A radiation polymerizable protective coating composition or paint, coated articles bearing such a protective radiation polymerizable paint which, on a non-polymerizable solvent, pigment, initiator and particulate filler-free basis, consists essentially of a binder solution of: (1) between about 90 and about 10 parts of a saturated, thermoplastic vinyl polymer prepared from at least about 85 weight percent of monofunctional vinyl monomers; (2) between about 10 and about 90 parts of vinyl solvent monomers for said vinyl polymer, at least about 10 weight percent, preferably at least about 30 weight percent, of said solvent monomers being selected from the group consisting of divinyl monomers, trivinyl monomers, tetravinyl monomers and mixtures thereof; and (3) between about 1.0 and about 15.0 parts per 100 parts of the total of said thermoplastic vinyl polymer and said vinyl solvent monomers of a triester of phosphoric acid bearing one or more sites of vinyl unsaturation. The composition exhibits excellent quality and good adhesion to a variety of substrates, in particular metals, including vapor deposited metals. Preferred articles bearing such a coating are prepared by: applying a base coat to a substrate and curing the same; vapor depositing a coating of metal over the surface of the base coat; and applying to and curing on the surface of the deposited metal the radiation polymerizable topcoat, preferably with little or no pigment contained therein.

7 Claims, No Drawings 


\section{Bibliografia}

Lux Research (2010) Business Wire. Liderazgo de los EE.UU se enfrenta a desafíos mundiales de nanotecnología. dice Lux. Agosto 19.

<http://www.electroiq.com/articles/stm/2010/08/us-nanotech-leadership. html>.

Salamanca-Buentello, F., Persad, D. L., Court, E.B., Martin D.K., Daar, A.S., Singer, P.A. (2005) Nanotechnology and the developing world. PLoS Medicine, vol. 2, núm. 4: 0300-0303.

Hullmann, A. (2006) The economic development of nanotechnology. An indicator based analysis. European Commission, DG Research. Unit "Nano S\&T - Convergent Science and Technologies: 3-34.

Court E., Daar A. S., Martin E., Acharya T., Singer P. A. (2004) Will Prince Charles diminish the opportunities of developing countries in nanotechnology? Nanotechweb.org News. 〈http//www.nanotechweb.org/articles/society/3>. Consultado en noviembre de 2011.

Lux Research (2005) Rush to market in nanosensors, but most aren't "nano”. Mayo.

Lux Research (2004) The Nanotech Report 2004.

Lux Research (2004) Sizing Nanotechnology's Value Chain. Octobre.

Huang, Z., Hsinchun, Ch, Lijun Y., Mihail C. Roco (2005) Longitudinal nanotechnology development (1991-2002): National Science Foundation funding and its impact on patents. Journal of Nanoparticle Research, 7: 343-376. 